\title{
Plan de marketing para el laboratorio de investigación de suelos y aguas como centro de producción competitivo
}

\author{
Marketing plan for the soil and water research laboratory as a competitive production \\ center
}

\author{
Cecibel Portocarrero Díaz, Ricardo Rafael Alva Cruz ${ }^{2}$
}

\begin{abstract}
RESUMEN
El objetivo de esta investigación fue proponer un plan de marketing para el Laboratorio de Investigación de Suelos y Aguas como centro de producción competitivo de la Universidad Nacional Toribio Rodríguez de Mendoza de Amazonas, con la finalidad de solventar los problemas actuales del laboratorio en aspectos como la oferta y la demanda. Se realizó esta investigación partiendo del hecho de que el laboratorio carece de un documento que le permita obtener posicionamiento en el mercado. La investigación parte de la formulación del siguiente problema: ¿Qué estrategias debe tener el plan de marketing del LABISAG como centro de producción competitivo de la UNTRM, Amazonas - 2015?, se tuvo como hipotesis que el plan de marketing se debe basar diagnóstico de análisis de la oferta y la demanda que le permita plantear propuestas para poder brindar un servicio competitivo y bajo los estándares requeridos y que contribuyan a la satisfacción de las necesidades de los usuarios. Se empleó el métodos inductivo y deductivo, el descriptivo y el analítico, propios de la investigación social del tipo descriptiva; esto permitió obtener como resultados un estudio de mercado para el laboratorio y que sirvió como base para la elaboración de la propuesta de plan de marketing. De la investigación se puede concluir que el plan de marketing ha sido diseñado para la solución de problemas relacionados con el servicio, posicionamiento, promoción, ventas y precios del Laboratorio de Investigación de Suelos y Aguas de la UNTRM.
\end{abstract}

Palabras clave: competitividad, posicionamiento, oferta, demanda, mercado.

\begin{abstract}
The objective of this research was to propose a marketing plan for the Soil and Water Research Laboratory as a competitive production centre of the Toribio Rodríguez de Mendoza of Amazonas National University, in order to solve the current problems of the laboratory in aspects such as supply and demand. This research was carried out based on the fact that the laboratory lacks a document that allows it to obtain market positioning. The investigation starts from the formulation of the following problem: What strategies should the marketing plan of the LABISAG have as the centre of competitive production of the UNTRM, Amazonas - 2015?, Was the hypothesis that the marketing plan It is necessary to analyze the supply and demand that allows you to propose proposals to provide a competitive service and under the required standards and to contribute to the satisfaction of the needs of users. We used the inductive and deductive methods, the descriptive and the analytical, typical of social research of the descriptive type; this allowed us to obtain as a result a market study for the laboratory that served as the basis for the preparation of the marketing plan proposal. From the research, it can be concluded that the marketing plan has been designed to solve problems related to the service, positioning, promotion, sales and prices of the Soil and Water Research Laboratory of the UNTRM.
\end{abstract}

Keywords: competitiveness, positioning, supply, demand, market.

${ }^{1}$ Bachiller en Turismo y Administración, cecypd@hotmail.com

${ }^{2}$ M. Sc. en gestión ambiental, rafatur18@hotmail.com 


\section{INTRODUCCIÓN}

Las universidades a nivel mundial, en la actualidad son constituidas y ejercen su razón de ser, bajo tres pilares fundamentales que son: la formación académica profesional, competitividad e investigación científica (Licona y Rangel, 2013). Bajo ese contexto, la Universidad Nacional Toribio Rodríguez de Mendoza de Amazonas crea el Laboratorio de Investigación de Suelos y Aguas (LABISAG), mediante el proyecto SNIP N ${ }^{\circ} 173852$ - "Construcción del Laboratorio de Investigación en Suelos y Aguas", el cual brinda apoyo al sector agrícola mediante la prestación del servicio de análisis de suelos y aguas, este laboratorio es un centro de producción de la universidad, por lo cual debe ser manejado bajo los enfoques empresariales competitivos (Puerto, 2010). De esta manera podrá desarrollar sus operaciones de manera sostenible en el tiempo y con un posicionamiento de mercado local, regional y nacional (Narváez y Fernández, 2007). Es por ello, que es necesario que cuente con un plan de marketing (García, 2013).

El marketing es uno de esos términos "mágicos" de los que los empresarios y gentes de empresa en general echan mano casi constantemente (Sainz, 2013).

En el ámbito internacional se desarrollaron investigaciones similares como la de López (2008) quien elaboró un plan de Marketing para el Laboratorio Clínico Patológico López Correa S.A., la investigación requirió un análisis del comportamiento del ambiente externo de la empresa desde el punto de vista económico, el sector salud en el país, la oferta que existen en el mercado (otros laboratorios) analizados desde el punto de vista de la competencia. Así mismo, Falquez et al. (2013) elaboró un plan estratégico de marketing interno para la industria Acromax S.A. laboratorio químico farmacéutico S.A. sucursal Guayaquil, partiendo del problema que Acromax S.A. posee una cultura organizacional distorsionada por la falta de canales de comunicación interna definidas y actividades de marketing interno y cultura organizacional.

En el ámbito nacional Quispe y Cipriano (2014) elaboraron un plan de marketing y estimación de su impacto en las ventas de la empresa turismo ejecutivo S.R.L. de la ciudad de Trujillo, encontraron como alternativa de solución propusieron la estrategia de Marketing Mix.

El presente trabajo de investigación pretende proponer un plan de Marketing para el Laboratorio de Investigación de Suelos y Aguas como centro de producción competitivo de la UNTRM basado en un diagnóstico de análisis de la oferta y la demanda que le permita plantear propuestas que mitiguen la problemática encontrada.

\section{MATERIAL Y MÉTODOS}

\section{a. Población, muestra y muestreo}

\section{- Población}

Se consideró como población a los clientes que hicieron uso de los servicios del LABISAG, que suman un total de 41 clientes, según la base de datos de clientes del Laboratorio, durante los años 2013 y 2014.

\section{-Muestra y muestreo}

Debido al tamaño de la población, se eligió considerar al total de la población y por ende no fue necesario hacer un muestreo.

\section{-Diseño de la investigación}

En cuanto al modelo experimental se usó el diseño con una muestra a la cual se le aplicó diferentes instrumentos y se obtuvo un diagnóstico del mercado en el que se desarrolla el Laboratorio de Investigación en Suelos y Aguas de la UNTRM.

\section{b.Métodos, técnicas e instrumentos de recolección de datos}

La tesis se fundamenta en los siguientes métodos:

Método inductivo y deductivo: Se realizó para el acopio de información, el cual fue el respaldo teórico científico que se fundamentó en el marco teórico (Dávila, 2006).

Método descriptivo: Se aplicó en el trabajo de campo y permitió que se registre información primaria que fue la base para el diagnóstico que requiere la investigación para poder elaborar el plan de marketing (Lafuente y Marín, 2008).

Método analítico: Facilitó el proceso de análisis de los resultados de la investigación (Lopera et al., 2010).

Las técnicas requeridas para la tesis son: observación directa, observación indirecta, técnica del fichaje, técnica de la encueta y técnica de la entrevista. Todas estas están orientadas a obtener información primaria de las poblaciones mencionadas en el punto que corresponde.

\section{Instrumentos}

Los instrumentos aplicados fueron la encuesta y la entrevista que fueron validados y estructurados teniendo en cuenta la obtención de la información de la oferta y la demanda que permitirían formular el plan de marketing de la tesis. 


\section{c. Análisis de datos}

La información se analizó mediante el método analítico y con el apoyo del software estadístico SPSS v.20.0. para realizar el análisis de frecuencia de cada una de las variables en estudio a partir de la base de datos de la encuesta. También se utilizó la técnica del cruce de información de diferentes fuentes primarias que permitió la contrastación de los resultados.

\section{RESULTADOS}

\section{Resultados del ambiente situacional}

\section{Características generales}

El Laboratorio de Investigación de Suelos y Agua de la UNTRM fue construido mediante el proyecto SNIP N 173852 "Construcción del Laboratorio de Investigación en Suelos y Agua de la UNTRM", del Instituto de Investigación para el Desarrollo Sustentable de Ceja de Selva (INDES-CES) de la Universidad Nacional Toribio Rodríguez de Mendoza. Los servicios como centro de producción se realizan partiendo de la necesidad de agricultores, asociaciones agrarias e instituciones de gobierno local en realizar estudios de suelos y aguas con fines diversos como el financiamiento económico de proyectos agrícolas o como parte de proyectos de desarrollo social que tienen los gobiernos locales.

\section{Perfil competitivo del servicio}

El LABISAG, posee un portafolio de servicios que son: Análisis de agua residuales, Análisis de agua, Análisis de suelo de rutina, Análisis físico químicos microbiológico y es ofertado entre nichos de mercado básicos que por su constitución son personas naturales, asociaciones agrarias e instituciones públicas y por su procedencia de carácter regional.

Según datos proporcionados por la oficina tesorería de la UNTRM. Según la distribución de los servicios prestados por el Laboratorio de Investigación de Suelos y Agua, el 61\% son análisis de suelo de rutina, el $32 \%$ análisis de agua, el 5\% análisis físico químico microbiológico, el 2\% análisis de aguas residuales.

Con datos proporcionados por la oficina de Tesorería de la UNTRM. Según el tipo de clientes el 60\% son personas naturales, el 26\% son asociaciones y empresas y el $14 \%$ son instituciones públicas. Las personas naturales son los principales clientes de los laboratorios debido a que estos de manera independiente son las que requieren de documentos de análisis de suelos para sus diferentes trámites relacionados con su actividad económica.
Los laboratorios de análisis de suelos y agua se concentran en $50 \%$ en Lima, teniendo en cuenta que este es una plaza de mercado amplia, incluso recibe clientes de las diferentes regiones del país. Los laboratorios de Lambayeque (17\%) se han posicionado de una parte de la región norte del país y sus principales clientes son agricultores. Como competencia directa al Laboratorio de Investigación de Suelos y Aguas de la UNTRM se podría considerar al laboratorio ubicado en Jaén ( $8 \%$ ) y al de Lambayeque por su cercanía a la región y teniendo en cuenta que estos eran utilizados anteriormente por los clientes de la región Amazonas. Se debe resaltar también que en Lima se encuentra el Laboratorio de Análisis de Agua, Suelo y Medio Ambiente UNALM, que es un centro de producción universitario como el LABISAG, materia de esta investigación.

El liderazgo que viene ejerciendo el LABISAG se basa en los siguientes aspectos:

- Forma parte de un instituto de investigación de la UNTRM

- Su equipamiento tecnológico es moderno y de punta

- Cuenta con recurso humano calificado y competente

\section{Barreras de salida}

La inversión en equipos, materiales y reactivos iniciales, ha sido parte del proceso del proyecto SNIP $\mathrm{N}^{\circ} 173852$, puede contribuir a reducir la dificultad de salida del sector, por otro lado, son equipos especializados y se complica su venta posterior.

\section{Poder de los competidores}

Este sector tiene un dinamismo medio en el poder de los compradores debido a que en muchos casos estos servicios son de exigencia obligatoria para la realización de proyectos y financiamiento de actividades. Los competidores se encuentran en otras partes del país, siendo los de mejor calidad los del departamento de Lima, entre ellos el Laboratorio de Análisis de Agua, Suelo y Medio Ambiente de la UNALM.

\section{Productos sustitutos}

Existen kits de análisis que podrían ser utilizados por los clientes para realizar pruebas directamente en campo. Existe una tendencia a incrementar su uso a nivel mundial. Esto no sustituye la calidad de los resultados del laboratorio de suelos y agua de la UNTRM.

\section{Análisis de las capacidades competitivas del LABISAG}

En esta etapa se identificaron las fortalezas, 
debilidades, oportunidades y amenazas que presenta el Laboratorio de Investigación en Suelos y Aguas, con el fin de contar con un punto de partida para la elaboración del plan de marketing.

\section{Estudios sobre la demanda del mercado}

El estudio general sobre la demanda del mercado contuvo los siguientes resultados:

- Definición del negocio

- Definición del mercado

- Análisis de la competencia directa más cercana

- Resultados de los ámbitos de consumo de los clientes de los laboratorios

Para determinar los hábitos de consumo de los clientes del Laboratorio de Investigación de Suelos y Aguas de la UNTRM. Se aplicó una encuesta a 41 personas, se obtuvo los siguientes resultados:

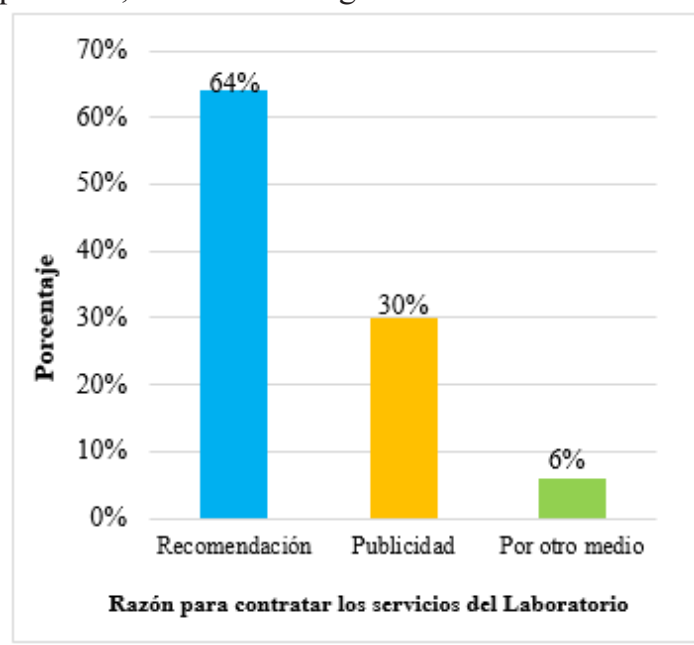

Figura $\mathrm{N}^{\circ}$ 01: Distribución de clientes según como llego a contratar los servicios del laboratorio

La preferencia de los usuarios del Laboratorio de Investigación de Suelos y Aguas está determinada por la veracidad en un $90 \%$, precio en un $80 \%$, rapidez en un $60 \%$ y el $48 \%$ por la asesoría técnica.

Según la encuesta, las fortalezas del laboratorio son contar con tecnología de punta $(42 \%)$, pertenecer a la universidad (32\%), pertenecer al INDES-CES (26\%). Entre las debilidades del laboratorio están: no ser muy conocido (48\%), no existe publicidad (46\%) y otros $(6 \%)$. Sus debilidades están ligadas a los procesos administrativos específicamente relacionados con el marketing y promoción que debe tener este centro de producción para poder incrementar su rentabilidad y productividad. Según la atención brindada por el personal el $78 \%$ es muy bueno y el $22 \%$ regular. La opinión al trato por lo general es favorable existiendo un déficit de menos del $30 \%$ que se debería de mitigar con estrategias incluidas dentro de un plan de marketing.

\section{Precio}

Considerando que el precio es un factor crítico en la dicción de tomar el servicio, el laboratorio ha establecido sus precios en relación al promedio de los precios del mercado. Los clientes emiten la siguiente opinión sobre el precio: La distribución de la población según costo de servicios es del 64\% que consideran que el precio está acorde al servicio, el $27 \%$ lo considera barato y el $9 \%$ caro. Existe una satisfacción casi generalizada por el pago de los servicios considerándole que esta es acorde al trabajo que se realiza.

\section{Promoción}

La forma de promoción es limitada a través de trípticos que son distribuidos en las ferias a nivel local, regional y en algunos casos nacionales.

De la entrevista realizada al Director Ejecutivo del INDES-CES, M. Sc. Segundo Manuel Oliva Cruz, se puede concluir que existe una proyección planificada a futuro y que el Laboratorio de Investigación de Suelos y Aguas tiene como visión ser un laboratorio líder en el Nor-oriente del país, brindando servicios para el desarrollo de investigaciones y mejorar la producción agropecuaria y contribuir al manejo eficiente de los recursos naturales.

La administración técnica del Laboratorio de Investigación de Suelo y Aguas lo realiza el INDESCES y su estado es rentable debido al dinamismo de producción de servicios que existe y sus líneas de productos variables.

\section{Resultados del plan de marketing}

El plan se compone de los siguientes aspectos:

Visión: Ser un Laboratorio de Investigación de Suelos y Aguas líder del nororiente peruano, brindando servicios para el desarrollo de investigaciones, para mejorar la producción agropecuaria y contribuir al manejo eficiente de los recursos naturales.

Misión: Somos un laboratorio dedicado a contribuir al desarrollo del sector agrícola y ganadero, a través de los servicios que brindamos, atendiendo las necesidades de nuestros clientes en forma eficiente y oportuna, basados en los principios de responsabilidad y transparencia; con talento humano profesional capacitado y tecnología avanzada, garantizando así altos estándares de calidad.

Oportunidad: El laboratorio tiene como oportunidades aprovechar la importancia que se le está dando al desarrollo de la agricultura y ganadería 
a nivel nacional, macro regional y regional, así mismo se está promoviendo la investigación científica en estos campos; este le permitirá mantener los servicios en el mercado y el poder ampliar a otros servicios de acuerdo a las exigencias del cliente.

Situación: El Laboratorio de Investigación de Suelos y Aguas se encuentra bajo la administración del INDES-CES, tiene una construcción específica para sus fines, su personal es con experiencia y capacitado, tiene una cartera de clientes establecida, sus canales de distribución no están claros y establecidos y a pesar de ello como centro de producción es rentable.

Objetivo de crecimiento: Establecer un crecimiento continuo anualmente llegando a abarcar el $70 \%$ del mercado local y el $20 \%$ del mercado macro regional.

\section{Marketing estratégico}

Perfil del consumidor de los servicios: Basado en el diagnostico mostrado en el capítulo anterior, el consumidor tiene el siguiente perfil:

- Su medio de información es directo o por recomendación de otros usuarios.

- Utiliza más el servicio de análisis de suelos de rutina y análisis de agua.

- La necesidad de contratar los servicios que brinda el Laboratorio de Investigación de Suelos y Aguas es obligatoria, en los fines que persigue.

- Tienen una opinión favorable referente a los servicios de laboratorio, ven como una fortaleza la tecnología y equipamiento del laboratorio y otro considera una debilidad en los inadecuados canales de distribución.

Deseos y necesidades de los consumidores: De acuerdo a los resultados de encuestas a los clientes, estos consideran 4 aspectos fundamentales para contratar el servicio: rapidez, asesoría técnica, precio, veracidad de los resultados del análisis de las muestras.

Mercado: son agricultores y ganaderos locales, regionales, macro regionales, asociaciones e instituciones públicas y privadas, investigadores, alumnos de pregrado y post grado, entre otros.

Estacionalidad: Según los datos obtenidos en la oficina de tesorería de la UNTRM, la estacionalidad de la demanda del servició es equilibrada no existiendo un mercado despuente de esta en un mes específico.

Competidores: Los competidores han sido identificados y están detallados en el estudio de mercado previo y por las características organizacionales se considera a un competidor importante, el Laboratorio de Análisis de Agua, Suelos y Medio Ambiente de la UNALM.

\section{Promoción y venta}

\section{Características de la promoción}

El Laboratorio de Investigación de Suelos y Aguas de la UNTRM, no posee estrategias claramente definidas para la promoción de los servicios, cada uno de ellos tiene sus propias estrategias y el posicionamiento en el mercado lo consiguen por recomendación boca a boca por parte de los clientes.

\section{Relaciones públicas}

Estas actividades deben formar parte de las acciones planificadas del INDES-CES, que tiene la administración de este centro de producción y deben realizarse visitas a los clientes más importantes, proporcionar información de las investigaciones y otras acciones que les tenga en contacto directo con el laboratorio.

\section{Aspectos legales}

E1 LABISAG forma parte del Instituto de Investigación para el Desarrollo Sustentable de Ceja de Selva de la UNTRM y se debe regir de acuerdo a lo normado en los reglamentos de este instituto, el estatuto de la universidad y la ley universitaria.

Precios: los precios son establecidos de acuerdo a criterios técnicos que se establecen en el instituto, y son registrados en el TUPA de manera anual.

\section{Marketing táctico}

\section{a) Servicios}

En el sector analizado el servicio de análisis de suelos y agua es un servicio nuevo y de crecimiento progresivo; el Laboratorio de Investigación de Suelos y Aguas de la UNTRM propone diferenciarse con la oferta de los mejores tiempos de respuesta, asesoría técnica post venta y cálida en el informe de resultados.

\section{b) Beneficios para el consumidor}

La fertilización de los cultivos tanto en exceso como en defecto reduce la cálida de las cosechas y los beneficios económicos, los análisis de suelos y agua son la mejor herramienta para evaluar la fertilidad del suelo a fin de darles el mejor aprovechamiento.

\section{c) Marca}

El concepto de marca del laboratorio debe estar en que forma parte de un instituto y una universidad de prestigio de la macro región. 


\section{d) Diseño}

Este ha sido concebido de acuerdo a los lineamientos de marketing que le permitirán al Laboratorio de Investigación de Suelos y Aguas de la UNTRM ser más competitivo.

\section{e) Proceso del plan de marketing}

El proceso parte de identificar las oportunidades del servicio que tiene el laboratorio, dentro del mercado que se desarrolla y bajo las características por las que fue concebido considerando las necesidades que en este caso tienen las instituciones públicas, asociaciones privadas, investigadores, estudiantes, agricultores particulares que forman el componente demanda y que se ubican en el ámbito local, regional y nacional.

En la segunda parte se determinó los elementos de entrada es decir parte del registro de la información de una manera sistematizada que van a permitir realizar análisis que se aplican al nuevo diseño teniendo en cuenta las necesidades, los recursos tantos físicos, humanos, los reglamentos y aspectos legales y otros requisitos necesarios para la ejecución de un plan de marketing.

En una tercera etapa se analizó la factibilidad para la implementación de esta donde se exige la respuesta que debe ser aprobatoria y que permite la continuación y aplicación del plan en un tiempo y espacio determinado

\section{f) Calidad}

Este factor en el laboratorio está garantizado partiendo de dos componentes que son la tecnología y equipamiento y en recurso humano con capacidades y actitudes adecuadas para brindar el servicio desde el aspecto técnico y de la atención al cliente.

\section{g) Punto de venta}

\section{Canales de distribución}

El laboratorio no vende a través de canales indirectos, todos los clientes deben ser directos, por las características que presenta el servicio de análisis de suelo y agua y que los elementos indirectos podrían repercutir en la cálida de servicio.

\section{h) Promoción}

\section{Publicidad}

Considerando que el desconocimiento del laboratorio influye en la cantidad de servicios que presta, necesitan hacer conocer su existencia y productos ofertados, para incrementar sus ventas y posición en el mercado.

\section{i) Estrategia}

Para realizar la publicidad debe basarse en las fuentes principales a través de las cuales el cliente se entera de la existencia del laboratorio y que son de transmisión directa, por recomendación, la publicidad impresa en trípticos, volantes, dípticos y también se debe incrementar su inclusión en el internet, revistas y otros medios masivos., incrementar su participación en ferias y eventos con asistencia masiva.

\section{i) Medios de comunicación}

\section{- Objetivo}

Dar a conocer el laboratorio a los diferentes segmentos del mercado con los que trabaja.

\section{- Estrategia}

Se debe de utilizar como estrategia las notas de prensa, conferencias, entrevistas y reportajes tanto en medios locales, regionales y nacionales.

\section{- Herramientas y promoción de venta}

Se diseñará un catálogo de portafolios de servicios por segmento objetivo. Se diseñarán cuatro promociones al año con el fin de implementar la demanda y estas podrán ser: por la compra de un análisis que podría incluir asesoría técnica gratuita o con un porcentaje de descuento.

\section{j) Precio}

Los precios del laboratorio serán acordes a los estándares del mercado, sin reducirlos o excederlos, la estrategia está en competir en la calidad y no en el costo del servicio.

\section{k) Márgenes de ganancia}

El margen neto debe ser del 30\%.

\section{DISCUSIÓN}

Quispe y Cipriano (2014) presentan una investigación prospectiva, destinada a medir el impacto de un plan de marketing, pero para un rubro diferente; la presente investigación tiene como objeto de estudio al Laboratorio de Investigación de Suelos y Aguas, donde la demanda de mercado es más reducida y especializada por tanto las estrategias de plan de marketing deben ser adaptadas esta realidad.

Frente a lo expuesto en las investigaciones de López (2008), Falquez y Zambrano (2013) y Valdivieso (2014) y lo obtenido en la presente investigación se puede discutir que los planes de marketing tienen como objetivo buscar un posicionamiento en el mercado y el incremento de los clientes quienes permiten la vida empresarial. La diferencia entre estas investigaciones y la presente investigación 
radica en los siguientes aspectos: el primero que el tipo de servicio es diferente, uno ligado a la salud y en esta investigación ligado a la agricultura, ganadería y afines que requieran análisis de suelos y agua; esta diferencia es importante resaltar considerando que por la amplitud del mercado de salud un laboratorio clínico presenta mayor dinamismo en el servicio, mientras que un laboratorio de suelo y agua tiene un segmento más especializado y científico, por tanto es más reducido y las estrategias que se formulen en su plan de marketing deben ser consideradas estas características; también existen diferencias en la estructura organizacional, el primer laboratorio es una empresa privada independiente y el Laboratorio de Investigación de Suelos y Aguas materia de esta investigación, funciona como un centro de producción que forma parte del Instituto de Investigación para el Desarrollo Sustentable de Ceja de Selva perteneciente a la UNTRM, y su administración y funcionamiento depende de ese orden jerárquico.

Tomala (2012) parte de la cultura institucional de la empresa que presenta indicadores de deficiencia, por lo que la investigación se orienta a un plan de marketing interno, en cambio en esta investigación el posicionamiento en el mercado es un problema a solucionar. La presente investigación también contempla lineamientos como la mejora de capacidades que están ligadas al marketing interno.

Lo discutido hasta el momento nos permitió la constatación y comprobación de la Hipótesis que se planteó en la investigación: que la propuesta del plan de marketing para el laboratorio debe ser basado en un diagnóstico de análisis de la oferta y la demanda que le permitan mitigar la problemática encontrada.

\section{CONCLUSIONES}

- Luego de realizar el diagnóstico del mercado en el que se desarrolla el LABISAG, se concluyó que al ser el único laboratorio de Amazonas que presta el servicio de análisis de suelos con fines agrícolas y debido a que esta región tiene como principales actividades económicas la agricultura y la ganadería, esto le ofrece la posibilidad de posicionarse como el mejor laboratorio de suelos y aguas de la región y del país, sin embargo tiene un fuerte competidor: el Laboratorio de Análisis de Suelo, Agua y Medio Ambiente de la Universidad Nacional Agraria La Molina como competencia directa por su constitución, tipos de organización y servicios brindados. Es por ello que se hace necesario buscar una buena estrategia de promoción y difusión para ampliar su mercado.

- Las principales potencialidades competitivas del
Laboratorio de Investigación de Suelos y Aguas de la UNTRM, radican en la tecnología y equipamiento de punta, recursos humanos, ubicación dentro del segmento y sus costes que van de acuerdo al tipo de servicios. Sus debilidades son: los canales de distribución utilizados en el proceso de comercialización y promoción, esta información se utilizó como base para la elaboración del plan de marketing del laboratorio.

- Las estrategias que se formularon para convertir al Laboratorio de Investigación de Suelos y Aguas como centro de producción competitivo fueron: el marketing estratégico y el marketing táctico

La propuesta del plan de marketing permitió establecer la visión, misión y objetivos de crecimiento del laboratorio en el mercado local, en un $70 \%$ y $20 \%$ en el ámbito macro regional estableciendo lineamientos de promoción y ventas que convertirán al laboratorio en un centro de producción competitivo a nivel regional y nacional.

\section{REFERENCIAS BIBLIOGRÁFICAS}

Dávila, G. (2006). El razonamiento inductivo y deductivo dentro del proceso investigativo en ciencias experimentales y sociales. LaurusRevista de Educación 12: 180-205.

Falquez, L. L., Zambrano, K. I. (2013). Plan estratégico de marketing interno para la industria Acromax laboratorio químico farmacéutico S. A., sucursal Guayaquil. Carrera de Administración de empresas, Universidad Politécnica Salesiana. Guayaquil, Ecuador, $101 \mathrm{pp}$

García, E. (2013). Diseño de plan de marketing estratégico caso: Hotel restaurant el Castillo. Trabajo de fin de grado. Faculta de ciencias sociales, jurídicas y de la comunicación, Universidad de Valladolid. Segovia, España. 64 pp.

Lafuente, C., Marí, A. (2008). Metodologías de la investigación en las ciencias sociales: Fases, fuentes y selección de técnicas. Revista Escuela de Administración de Negocios 64: 5-18.

Licona, A., Rangel, J. E. (2013). Pilares de la competitividad, educación superior, nuevas tecnologías y empleo en Corea del Sur y México. Análisis económico 69 (28): 79108.

Lopera, J. D., Ramírez, C. A., Ucaris, M., Ortiz, J. (2010). El método analítico. Revista de psicología 2 (4): 87-90. 
López, D. C. (2008). Plan de Marketing para el Laboratorio Clínico Patológico López Correa S.A. a diciembre de 2008. Facultad de Ciencias Económicas y Administrativas. Pontifica Universidad Javeriana. Bogotá, Colombia. 148 pp.

Narváez, M., Fernández, G. (2007). Estrategias competitivas para fortalecer sectores de actividad empresarial en el mercado global. Revista Venezolana de Gerencia 13 (42): 233-243.

Puerto, D. P. (2010). La globalización y el crecimiento empresarial a través de estrategias de internacionalización. Pensamiento y gestión (28): 171-195.

Quispe, M., Cipriano, S. (2014). Plan de marketing y estimación de su impacto en las ventas de la empresa turismo ejecutivo S.R.L. de la ciudad de trujillo-2014. Facultad de Ciencias Económicas, Universidad Privada Antenor Orrego. Trujillo, Perú. 143 pp.

Valdivieso, I. G. (2014). Plan de marketing para laboratorios Bagó, agencia Loja. Carrera de Administración de Empresas, Universidad Nacional de Loja. Loja, Ecuador. 187 pp. 